\title{
REPRESENTATION OF A BINARY QUADRATIC FORM AS A SUM OF TWO SQUARES
}

\author{
KENNETH S. WILLIAMS
}

\begin{abstract}
Let $\phi(x, y)$ be an integral binary quadratic form. A short proof is given of Pall's formula for the number of representations of $\phi(x, y)$ as the sum of squares of two integral linear forms.
\end{abstract}

Let $\phi(x, y)$ be an integral binary quadratic form. If $\phi(x, y)$ is expressible as the sum of squares of two integral linear forms then $\phi(x, y)$ must be positive definite or semidefinite, have an even coefficient of $x y$, and be of square determinant. Mordell [1] has proved that a binary quadratic form $\phi(x, y)=h x^{2}+2 k x y+l y^{2}$ with these properties is the sum of squares of two integral linear forms if and only if $r_{2}\left(d_{1}\right)>0$, where $r_{2}\left(d_{1}\right)$ denotes the number of representations of $d_{1}=$ G.C.D. $(h, 2 k, l)$ as the sum of two squares. Pall [2], using properties of Hermite-matrices, has shown that when $\phi(x, y)$ is representable in this way, the number of such representations is $2 r_{2}\left(d_{1}\right)$, if $\operatorname{det}(\phi)=h l-k^{2}=m^{2} \neq 0$, and is $r_{2}\left(d_{1}\right)$, if $\operatorname{det}(\phi)=h l-k^{2}=$ $m^{2}=0$. In this note we give a very simple proof of this result.

Since $h x^{2}+2 k x y+l y^{2}$ can be expressed as the sum of squares of two integral linear forms there exist integers $a_{1}, a_{2}, b_{1}, b_{2}$, such that

$$
h x^{2}+2 k x y+l y^{2}=\left(a_{1} x+b_{1} y\right)^{2}+\left(a_{2} x+b_{2} y\right)^{2} .
$$

If we write $\alpha, \beta$ for the gaussian integers $a_{1}+i a_{2}, b_{1}+i b_{2}$ respectively, (1) becomes

$$
h x^{2}+2 k x y+l y^{2}=(\alpha x+\beta y)(\bar{\alpha} x+\bar{\beta} y),
$$

so that

$$
h=\alpha \bar{\alpha}, \quad 2 k=\alpha \bar{\beta}+\bar{\alpha} \beta, \quad l=\beta \bar{\beta} .
$$

The domain of all gaussian integers is denoted by $Z(i)$. It is a unique factorization domain. We let $\gamma \in Z(i)$ denote one of the four associated greatest common divisors of $\alpha$ and $\beta$ and write $\alpha=\gamma \alpha_{1}, \beta=\gamma \beta_{1}$, so that the only common factors of $\alpha_{1}$ and $\beta_{1}$ are the units $\pm 1, \pm i$. Hence from

Received by the editors December 23, 1970.

AMS 1970 subject classifications. Primary 10C05, 10J05; Secondary 10E25.

Key words and phrases. Binary quadratic form, sum of squares.

(c) American Mathematical Society 1972 
(2) we have

that is

$$
\begin{aligned}
d_{1} & =\text { G.C.D. }(h, 2 k, l)=\text { G.C.D. }(\alpha \bar{\alpha}, \alpha \bar{\beta}+\bar{\alpha} \beta, \beta \bar{\beta}) \\
& =\gamma \bar{\gamma} \text { G.C.D. }\left(\alpha_{1} \bar{\alpha}_{1}, \alpha_{1} \bar{\beta}_{1}+\bar{\alpha}_{1} \beta_{1}, \beta_{1} \bar{\beta}_{1}\right),
\end{aligned}
$$

$$
d_{1}=\gamma \bar{\gamma}
$$

and so (2) becomes

$$
h x^{2}+2 k x y+l y^{2}=d_{1}\left(\alpha_{1} x+\beta_{1} y\right)\left(\bar{\alpha}_{1} x+\bar{\beta}_{1} y\right) .
$$

Moreover we have

that is,

$$
\begin{aligned}
m^{2} & =\operatorname{det}\left(h x^{2}+2 k x y+l y^{2}\right)=h l-k^{2} \\
& =(\alpha \bar{\alpha})(\beta \bar{\beta})-\left(\frac{\alpha \bar{\beta}+\bar{\alpha} \beta}{2}\right)^{2} \quad(\text { from }(3)) \\
& =-\left(\frac{\alpha \bar{\beta}-\bar{\alpha} \beta}{2}\right)^{2}=-(\gamma \bar{\gamma})^{2}\left(\frac{\alpha_{1} \bar{\beta}_{1}-\bar{\alpha}_{1} \beta_{1}}{2}\right)^{2} \\
& =-d_{1}^{2}\left(\frac{\alpha_{1} \bar{\beta}_{1}-\bar{\alpha}_{1} \beta_{1}}{2}\right)^{2} \quad(\text { from }(4)),
\end{aligned}
$$

$$
m= \pm d_{1} i\left(\frac{\alpha_{1} \bar{\beta}_{1}-\bar{\alpha}_{1} \beta_{1}}{2}\right)
$$

Now the required number of representations is just the number of distinct 4-tuples of integers $\left(a_{1}^{\prime}, a_{2}^{\prime}, b_{1}^{\prime}, b_{2}^{\prime}\right)$ such that

$$
h x^{2}+2 k x y+l y^{2}=\left(a_{1}^{\prime} x+b_{1}^{\prime} y\right)^{2}+\left(a_{2}^{\prime} x+b_{2}^{\prime} y\right)^{2},
$$

that is, on writing $\alpha^{\prime}=a_{1}^{\prime}+i b_{1}^{\prime} \in Z(i), \beta^{\prime}=a_{2}^{\prime}+i b_{2}^{\prime} \in Z(i)$ and using (5), the number of distinct pairs of gaussian integers $\left(\alpha^{\prime}, \beta^{\prime}\right)$ such that

$$
\left(\alpha^{\prime} x+\beta^{\prime} y\right)\left(\bar{\alpha}^{\prime} x+\bar{\beta}^{\prime} y\right)=d_{1}\left(\alpha_{1} x+\beta_{1} y\right)\left(\bar{\alpha}_{1} x+\bar{\beta}_{1} y\right) .
$$

As $\alpha_{1} x+\beta_{1} y$ is a primitive irreducible element in the unique factorization domain $Z(i)[x, y]$ we have

$$
\alpha_{1} x+\beta_{1} y \mid \alpha^{\prime} x+\beta^{\prime} y \text { or } \alpha_{1} x+\beta_{1} y \mid \bar{\alpha}^{\prime} x+\bar{\beta}^{\prime} y .
$$

If $\alpha_{1} x+\beta_{1} y \mid \alpha^{\prime} x+\beta^{\prime} y$ then $\alpha^{\prime} x+\beta^{\prime} y=\delta\left(\alpha_{1} x+\beta_{1} y\right)$ for some $\delta \in Z(i)$, and so we have

$$
\left(\alpha^{\prime}, \beta^{\prime}\right)=\left(\delta \alpha_{1}, \delta \beta_{1}\right), \quad \text { where } \delta \bar{\delta}=d_{1} .
$$

Similarly if $\alpha_{1} x+\beta_{1} y \mid \bar{\alpha}^{\prime} x+\bar{\beta}^{\prime} y$ we have

$$
\left(\alpha^{\prime}, \beta^{\prime}\right)=\left(\delta^{\prime} \bar{\alpha}_{1}, \delta^{\prime} \bar{\beta}_{1}\right), \quad \text { where } \delta^{\prime} \bar{\delta}^{\prime}=d_{1} .
$$


If $m \neq 0$, so that from (6) we have $\alpha_{1} \bar{\beta}_{1} \neq \bar{\alpha}_{1} \beta_{1}$, then $\left(\delta \alpha_{1}, \delta \beta_{1}\right) \neq\left(\delta^{\prime} \bar{\alpha}_{1}, \delta^{\prime} \bar{\beta}_{1}\right)$ and so (7) and (8) give $2 r_{2}\left(d_{1}\right)$ distinct pairs $\left(\alpha^{\prime}, \beta^{\prime}\right)$ as required. If $m=0$, so that from (6) we have $\alpha_{1} \bar{\beta}_{1}=\bar{\alpha}_{1} \beta_{1}$, then $\bar{\alpha}_{1} \sim \alpha_{1}, \bar{\beta}_{1} \sim \beta_{1}$ and the set of ordered pairs given by (7) coincides with that given by (8), thus giving only $r_{2}\left(d_{1}\right)$ distinct pairs $\left(\alpha^{\prime}, \beta^{\prime}\right)$ as required.

\section{REFERENCES}

1. L. J. Mordell, On the representation of a binary quadratic form as a sum of squares of linear forms, Math. Z. 35 (1932), 1-15.

2. G. Pall, Sums of two squares in a quadratic field, Duke Math. J. 18 (1951), 399-409. MR 12, 676.

Department of Mathematics, Carleton University, Ottawa, Ontario, Canada 